\title{
THE LEBESGUE DECOMPOSITION THEOREM FOR PARTIALLY ORDERED SEMIGROUP.VALUED MEASURES
}

\author{
PANAIOTIS K. PAVLAKOS
}

\begin{abstract}
The present paper is concerned with partially ordered semigroup-valued measures. Below are given generalizations of the classical Lebesgue Decomposition Theorem.

These results can be applied to Stone or $W^{*}$ algebra-valued positive measures (cf. [3], [12], [13], [14]).
\end{abstract}

1. Preliminaries. By a partially ordered semigroup $X$ we mean a commutative semigroup with identity 0 , equipped by a partial ordering $\leqslant$, compatible with the structure of $X$ under the conditions:

(i) If $x, y, z$ are elements of $X$ with $x<y(x \leqslant y$ and $x \neq y)$ then $x+z<y+z$.

(ii) $x+\sup E=\sup (x+E)$, whenever there exist sup $E$ (the supremum of $E$ in $X)$ and $\sup (x+E), E \subseteq X, x \in X$.

Now $X$ is monotone complete if every majorised increasing directed family in $X$ has a supremum in $X$. Moreover, $X$ is of the countable type if every subset $E$ of $X$ that has a supremum in $X$, contains a countable subset $E^{*} \subseteq E$ so that: $\sup E=\sup E^{*}$.

Let $X$ be a partially ordered semigroup and $H$ a ring of subsets of $T$. The function $m: H \rightarrow X$ is an $o$-measure (order measure) on $H$, if $m$ is positive on $H(m(A) \geqslant 0$, for every $A$ in $H)$ and $m\left(\cup_{n \in N} A_{n}\right)=\sup \left\{\sum_{i=1}^{n} m\left(A_{i}\right): n \in\right.$ $N$ \} whenever $\left(A_{n}\right)_{n \in N}$ is a disjoint sequence of elements of $H$ with $\left(\cup_{n \in N} A_{n}\right)$ $\in H$.

The following propositions can be easily proved.

Proposition 1.1. Let $m: H \rightarrow X$ be an o-measure on $H$.

(1) $m(\varnothing)=0$.

(2) $m$ is finitely additive on $H$ and $m(A) \leqslant m(B)$, whenever $A, B \in H$ with $A \subseteq B$.

(3) For every sequence $\left(A_{n}\right)_{n \in N}$ in $H$ with $\left(\cup_{n \in N} A_{n}\right) \in H$ and $\sup \left\{\sum_{i=1}^{n} m\left(A_{i}\right): n \in N\right\} \in X$, implies: $m\left(\cup_{n \in N} A_{n}\right) \leqslant \sup \left\{\sum_{i=1}^{n} m\left(A_{i}\right): n \in\right.$ $N\}$.

Received by the editors August 22, 1977 and, in revised form, November 30, 1977.

AMS (MOS) subject classifications (1970). Primary 46G99; Secondary 28A55.

Key words and phrases. Partially ordered semigroup, monotone complete partially ordered semigroup, partially ordered semigroup of the countable type, $o$-measure, absolutely continuous and singular $o$-measure, partially ordered topological semigroup, $\sigma$-compatible topology with the partial ordering, $\tau_{\boldsymbol{X}}$-measure. 
(4) If $X$ is monotone complete then for every disjoint family $\left(A_{i}\right)_{i \in I}$ in $H$ with $\left(\cup_{i \in I} A_{i}\right) \in H$ implies: $m\left(\cup_{i \in I} A_{i}\right) \geqslant \sum_{i \in I} m\left(A_{i}\right):=\sup \left\{\sum_{i \in J} m\left(A_{i}\right): J \subseteq I\right.$, $J$ finite $\}$.

Proposition 1.2. The function $m: H \rightarrow X$ is an o-measure on $H$ if and only if $m$ is positive, finitely additive on $H$ and $m\left(A_{n}\right) \uparrow m(A)\left(m\left(A_{n}\right) \leqslant m\left(A_{n+1}\right)\right.$, $n \in N$ and $\left.m(A)=\sup \left\{m\left(A_{n}\right): n \in N\right\}\right)$, for every increasing sequence $\left(A_{n}\right)_{n \in N}$ in $H$ with $A_{n} \uparrow A \in H$.

2. Absolutely continuous and singular $o$-measures. Let $X, Y$ be partially ordered semigroups and let $m: H \rightarrow X, l: H \rightarrow Y$ be $o$-measures on $H$. $l$ is $m$-absolutely continuous on $H(l \ll m)$ if $l(A)=0$ whenever $A \in H$ with $m(A)=0$. On the other hand $l$ is $m$-singular on $H,(l \perp m)$ if for every $A$ in $H$ there is $B$ in $H: B \subseteq A, m(B)=0$ and $l(A-B)=0$. So $m \perp l$ if and only if $l \perp m$.

The following proposition can be easily verified.

Proposition 2.1. Let $m: H \rightarrow X, l: H \rightarrow Y$ and $k: H \rightarrow Y$ be o-measures on $H$.

(1) If $l \perp m$ and $l \ll m$ then $l=0$.

(2) If $l \perp m$ and $k \ll m$ then $l \perp k$.

(3) $l \perp l$ if and only if $l=0$.

(4) If $m \perp l$ and $m \perp k$ then $m \perp(l+k)$.

(5) If $l \perp m$ and $k \perp m$ then $(l+k) \perp m$.

(6) If $X=Y$, and $l \leqslant m+k, l \perp m$ then $l \leqslant k$.

On the other hand the following lemma will be useful in the sequence.

LEMMA 2.2. Let $m_{i}: H \rightarrow X, i \in I$, be an increasing directed family of $o$-measures on $H$. Suppose, that $X$ is a monotone complete partially ordered semigroup and for every $A \in H$ there is $x$ in $X$ such that: $m_{i}(A) \leqslant x$, whenever $i \in I$. Then the function $m: H \rightarrow X, m(A)=\sup \left\{m_{i}(A): i \in I\right\}$ is an $o-$ measure on $H$.

Proof. Let $A, B \in H$ with $A \cap B=\varnothing$, so $m(A \cup B)=\sup \left\{m_{i}(A \cup B)\right.$ : $i \in I\}=\sup \left\{m_{i}(A)+m_{i}(B): i \in I\right\} \leqslant \sup \left\{m_{i}(A): i \in I\right\}+\sup \left\{m_{i}(B): i\right.$ $\in I\}=m(A)+m(B)$. Furthermore let $i, j$ be any pair of indices. Then there exist $h \in I$ such that, $h>i$ and $h>j$, hence $m_{i}(A)+m_{j}(B) \leqslant m_{h}(A)+$ $m_{h}(B)=m_{h}(A \cup B) \leqslant m(A \cup B)$, which implies $m(A)+m(B)=m(A \cup$ $B$ ), namely $m$ is finitely additive on $H$. Evidently $m(A) \leqslant m(B)$ whenever $A$, $B \in H$ with $A \subseteq B$.

Finally let $\left(A_{n}\right)_{n \in N}$ be a sequence in $H$ with $A_{n} \uparrow A \in H$. Then $m_{i}\left(A_{n}\right) \uparrow m_{i}(A)$, for every $i$ in $I$. Thus:

$$
\begin{aligned}
\sup \left\{m\left(A_{n}\right): n \in N\right\} & =\sup \left\{\sup \left\{m_{i}\left(A_{n}\right): i \in I\right\}: n \in N\right\}, \\
m(A) & =\sup \left\{\sup \left\{m_{i}\left(A_{n}\right): n \in N\right\}: i \in I\right\} .
\end{aligned}
$$

But $\left\{m_{i}\left(A_{n}\right): i \in I, n \in N\right\}=\cup_{i \in I}\left\{m_{i}\left(A_{n}\right): n \in N\right\}=\cup_{n \in N}\left\{m_{i}\left(A_{n}\right): i\right.$ $\in I\}$, hence 


$$
\begin{aligned}
\sup \left\{\sup \left\{m_{i}\left(A_{n}\right): i \in I\right\}: n \in N\right\} & =\sup \left\{\sup \left\{m_{i}\left(A_{n}\right): n \in N\right\}: i \in I\right\} \\
& =\sup \left\{m_{i}\left(A_{n}\right): i \in I, n \in N\right\}
\end{aligned}
$$

(cf. [11, p. 12, Theorem I.6.1]). Therefore by (1), (2) and (3) it follows that $m\left(A_{n}\right) \uparrow m(A)$ and the assertion follows from Proposition 1.2.

Hereafter by $S$ it is denoted a $\sigma$-ring of subsets of $T$.

Proposition 2.3. Let $m_{i}: S \rightarrow X, i \in I$ be an increasing directed family of $o$-measures on $S$ and $l: S \rightarrow Y$ be another o-measure on $S$. Suppose that $X$ is of the countable type partially ordered semigroup, $\sup \left\{m_{i}(A): i \in I\right\}=m(A) \in$ $X$, whenever $A \in S$ and $m_{i} \perp l$ for every $i \in I$. Then $m: S \rightarrow X$ is an 0 -measure on $S$ with $m \perp l$.

Proof. By Lemma 2.2 it follows that $m$ is an $o$-measure on $S$. Now let $A \in S$. Then there is a countable subset $\{i(n): n \in N\}$ of $I$, such that: $m(A)=\sup \left\{m_{i(n)}(A): n \in N\right\}$. On the other hand, there is a sequence $\left(B_{n}\right)_{n \in N}$ in $S$ with $B_{n} \subseteq A, m_{i(n)}(A)=m_{i(n)}\left(B_{n}\right)$ and $l\left(B_{n}\right)=0$, for every $n \in N$. We put $B=\cup_{n \in N} B_{n}$ hence $B \subseteq A, m_{i(n)}(A)=m_{i(n)}(B)$ and $l(B)=$ $0, n \in N$. Consequently

$$
\begin{aligned}
& m(A)=\sup \left\{m_{i(n)}(A): n \in N\right\}=\sup \left\{m_{i(n)}(B): n \in N\right\} \leqslant m(B) \leqslant m(A), \\
& \text { so } m(A-B)=0 \text { and } l(B)=0 .
\end{aligned}
$$

COROllary 2.4. Let $m_{n}: S \rightarrow X, n \in N$, be an increasing sequence of $o$-measures on $S$ and let $l: S \rightarrow Y$ be another o-measure on $S$. Suppose that $\sup \left\{m_{n}(A): n \in N\right\}=m(A) \in X$, whenever $A \in S$ and $m_{n} \perp l$, for every $n \in N$. Then $m: S \rightarrow X$ is an o-measure on $S$ and $m \perp l$.

3. The Lebesgue Decomposition Theorem. First we give the following:

LEMMA 3.1. Let $m: S \rightarrow X$ be an o-measure on the $\sigma$-ring $S$ and let $\Lambda$ be a nonempty subfamily of $S$ closed to countable unions. Suppose that $X$ is a monotone complete of the countable type partially ordered semigroup. Then the function $m_{1}: S \rightarrow X, m_{1}(A)=\sup \{m(A \cap M): M \in \Lambda\}$, is an o-measure on $S$ and for every $A$ in $S$, there exists $M \in \Lambda$ such that $m_{1}(A)=m(A \cap M)$.

Proof. Let $A \in S$. From the hypothesis it is easily verified that there exists an increasing sequence $\left(M_{n}\right)_{n \in N}$ in $\Lambda$ with $M_{n} \uparrow M \in \Lambda$ and $m(A \cap M)=$ $\sup \left\{m\left(A \cap M_{n}\right): n \in N\right\}=m_{1}(A)$.

Next let $\left(m_{M}\right)_{M \in \Lambda}$ be the increasing directed family of $o$-measures on $S$, such that $m_{M}(A)=m(A \cap M)$ whenever $M \in \Lambda$ and $A \in S$. By Lemma 2.2 and from $m_{1}(A)=\sup \left\{m_{M}(A): M \in \Lambda\right\}, A \in S$ it follows that $m_{1}$ is an $o$-measure on $S$.

Theorem 3.2 (Lebesgue Decomposition). Let the o-measures be $m: S \rightarrow$ $X, l: S \rightarrow Y$ on the $\sigma$-ring $S$. Suppose, $Y$ is a monotone complete of the countable type partially ordered semigroup. Then there exist unique o-measures $l_{i}: S \rightarrow Y, i=1,2$, such that: 


$$
l=l_{1}+l_{2}, \quad l_{1} \ll m, \quad l_{2} \perp m, \quad l_{1} \perp l_{2}
$$

Proof. We consider the functions: $l_{1}: S \rightarrow Y, l_{2}: S \rightarrow Y, l_{1}(A)=\sup \{l(A$ $\cap M): \quad M \in \Lambda\}, \quad l_{2}(A)=\sup \{l(A \cap Q): Q \in \Theta\}$ where $\Theta=\{Q \in S:$ $m(Q)=0\}$ and $\Lambda=\left\{M \in S: l_{2}(M)=0\right\}$. Clearly from Lemma 3.1 the functions $l_{i}: S \rightarrow Y, i=1,2$, are $o$-measures on $S$ and there exist $M \in \Lambda$, $Q \in \Theta$ such that:

$$
\begin{aligned}
& l_{1}(A)=l(A \cap M)=l_{1}(A \cap M), \\
& l_{2}(A)=l(A \cap Q)=l_{2}(A \cap Q) .
\end{aligned}
$$

If $m(A)=0$ then $(A \cap M) \in \Theta$, hence $l_{1}(A)=l(A \cap M)=l_{2}(A \cap M)=$ 0 , namely $l_{1} \ll m$.

On the other hand $(A-Q) \in \Lambda$ (because $(A-Q) \notin \Lambda$ implies $l_{2}(A-$ $Q)>0$, so by (5) $l_{2}(A)>l_{2}(A)$, that is a contradiction), therefore $l(A-Q)$ $=l_{1}(A-Q)=l_{1}(A)$. Thus $l(A)=l(A-Q)+l(A \cap Q)=l_{1}(A)+l_{2}(A)$. Now from (4) and (5) one obviously has $l_{1} \perp l_{2}$ and $l_{2} \perp m$. To show uniqueness let $l=l_{1}+l_{2}=l_{3}+l_{4}$ be two such decompositions. Evidently $l_{4} \perp l_{1}$ and $l_{2} \perp l_{3}$. So from $l_{2} \leqslant l_{3}+l_{4}$ and $l_{4} \leqslant l_{1}+l_{2}$ imply $l_{2} \leqslant l_{4}$ and $l_{4} \leqslant l_{2}$, hence $l_{2}=l_{4}$. Furthermore from $l_{1} \perp l_{2}, l_{3} \perp l_{2}, l_{1} \leqslant l_{2}+l_{3}$ and $l_{3} \leqslant l_{1}$ $+l_{2}$ we also have $l_{1}=l_{3}$.

4. Partially ordered topological semigroup-valued measures. Throughout this paragraph we suppose that $X$ is a partially ordered topological semigroup, that is a partially ordered semigroup, equipped with a Hausdorff topology $\tau_{X}$ such that the sets: $E_{x}:=\{y \in X: y \geqslant x\}, F_{x}:=\{y \in X: y \leqslant x\}$ are $\tau_{X^{-}}$ closed, whenever $x \in X$. In this place we give the well-known lemma.

LEMMA 4.1. Let $\left(x_{i}\right)_{i \in I}$ be an increasing directed family in the partially ordered topological semigroup $X$ with $\tau_{X}$-lim $x_{i}=x$ (convergence in the topology $\tau_{X}$ of $X$ ). Then $x=\sup \left\{x_{i}: i \in I\right\}$.

Proof. We set $E_{i}=\left\{y \in X: y \geqslant x_{i}\right\}$ for every $i \in I$, hence $x \in \bar{E}_{i}=E_{i}$ (by $\bar{E}_{i}$ we denote the closure of $E_{i}$ in $X$ ), namely $x \geqslant x_{i}$ for every $i \in I$. Moreover let $z$ be an element of $X$ so that:

$$
x_{i} \leqslant z, \text { for any } i \in I .
$$

Thus by the fact that the set $F=\{y \in X: y \leqslant z\}$ is $\tau_{X}$-closed and hypothesis, one similarly has, $x \in \bar{F}=F$, which proves the assertion. Next the topology $\tau_{X}$ is called $\sigma$-compatible with the partial ordering if every majorised increasing sequence in $X$ converges relative to the topology $\tau_{X}$.

Now the function $m: H \rightarrow X$ is a $\tau_{X}$-measure on the ring $H$, if $m$ is positive on $H$ and $m$ is $\sigma$-additive on $H$ with respect to topological convergence in $X$. The definitions and results of absolute continuity and singularity are similar as above.

In particular we obtain.

THEOREM 4.2. Let the $\tau_{X}$-measure $m: S \rightarrow X$ and the $\tau_{Y}$-measure $l: S \rightarrow Y$ on the o-ring $S$. Suppose that $Y$ is a monotone complete of the countable type 
partially ordered topological semigroup and the topology $\tau_{Y}$ is $\sigma$-compatible with the partial ordering. Then there exist unique $\tau_{Y}$-measures $l_{i}: S \rightarrow Y, i=1,2$, such that:

$$
l=l_{1}+l_{2}, \quad l_{1} \ll m, \quad l_{2} \perp m, \quad l_{1} \perp l_{2} .
$$

The proof of the Theorem 4.2 follows from Lemma 4.1 and Theorem 3.2.

\section{REFERENCES}

1. S. K. Berberian, Measure and integration, Macmillan, New York, 1965.

2. R. Cristescu, Integrarea in spatii semiordonate, Acad. Repub. Pop. Romine. Bul. Sti. Sect. Sti. Mat. Fiz. 4 (1952), 291-310.

3. W. Hackenbroch, Zum Radon-Nikodymschen Satz fur positive Vectormasse, Math. Ann. 206 (1973), 63-65.

4. P. R. Halmos, Measure theory, Van Nostrand, Princeton, N. J., 1950.

5. R. A. Johnson, On the Lebesgue decomposition theorem, Proc. Amer. Math. Soc. 18 (1967), 628-632.

6. P. K. Pavlakos, Measures and integrals in ordered topological groups, Thesis, University of Athens, 1972.

7. A. Peressini, Ordered topological vector spaces, Harper and Row, New York, 1967.

8. M. Sion, A theory of semigroup-valued measures, Lecture Notes in Math., vol. 355, Springer-Verlag, Berlin and New York, 1973.

9. T. Traynor, Differentiation of group-valued outer measures, Thesis, University of British Columbia, 1969.

10. , Decomposition of group-valued additive set functions, Ann. Inst. Fourier (Grenoble) 22 (1972), 131-140.

11. B. Z. Vulikh, Introduction to the theory of partially ordered spaces, Wolters-Noordhoff, Groningen, 1967.

12. M. J. D. Wright, Stone-algebra-valued measures and integrals, Proc. London Math. Soc. 19 (1969), 107-122.

13. __ Vector lattice measures on locally compact spaces, Math. Z. 120 (1971), 193-203.

14. __ Measures with values in a partially ordered vector space, Proc. London Math. Soc. 25 (1972), 675-688.

Department of Mathematical Analysis, Athens University, Panepistemiopolis, Athens 621, GreECE 